\title{
SIMPLIFICAÇÃO DA TÉCNICA PARA REABILITAÇÃO DE MAXILA ATRÓFICA COM IMPLANTES ZIGOMÁTICOS COM INTERFACE CÔNICA INTERNA E CARREGAMENTO IMEDIATO: RELATO DE CASO
}

\author{
SIMPLIFICATION OF THE TECHNIQUE FOR REHABILITATION OF ATROPHIC \\ UPPER JAW WITH ZYGOMATIC IMPLANTS WITH INTERNAL TAPERED \\ INTERFACE AND IMMEDIATE LOADING: CASE REPORT
}

\author{
Geninho THOMÉ1; Jean UHLENDORF2; Carolina Accorsi CARTELLI3; Sérgio Rocha BERNARDES4; Larissa Carvalho TROJAN5; \\ Marcos Boaventura de MOURA ${ }^{6}$; Luis Eduardo Marques PADOVAN ${ }^{7}$ \\ 1 - Doutor em Implantodontia pela Faculdade de Medicina e Odontologia São Leopoldo Mandic, Campinas - SP; \\ 2 - Mestre e Especialista em Implantodontia pelo Instituto Latino Americano de Pesquisa e Ensino Odontológico (ILAPEO), Curitiba - PR; \\ 3 - Mestre e Especialista em Implantodontia pelo ILAPEO, Curitiba -PR; \\ 4 - Doutor em Reabilitação Oral pela Universidade de São Paulo (USP). Especialista em Prótese Dentária pela Associação Paulista dos Cirurgiões- \\ Dentistas (APCD), Bauru-SP; \\ 5 - Doutora em Engenharia de Estruturas pela Universidade Federal de Minas Gerais (UFMG). Especialista em Ortodontia pelo ILAPEO, Curitiba - PR; \\ 6 - Doutorando em Clínica Odontológica área de Implantodontia e Prótese sobre implante pela Universidade Federal de Uberlândia (UFU). \\ Especialista em Implantodontia pela Faculdade de Medicina e Odontologia São Leopoldo Mandic, Brasília - DF; \\ 7 - Doutor em Odontologia pela Universidade Estadual Paulista Júlio de Mesquita Filho (UNESP), São Paulo - SP.
}

\section{RESUMO}

A reabilitação de maxilas atróficas com implantes dentários é sempre um desafio, visto que o uso de enxertos ósseos é quase sempre necessário para estabilização dos implantes. Os implantes zigomáticos têm sido utilizados como uma opção viável ao uso de procedimentos de enxertia óssea anterior à cirurgia. A interface cônica interna apresenta gap reduzido entre implante e componente protético, mostrando resultados biológicos e estéticos satisfatórios a longo prazo. Este artigo tem como objetivo relatar a reabilitação protética imediata de uma maxila atrófica com implantes convencionais e zigomáticos com interface cônica interna como opção ao uso de enxertos ósseos prévios. Paciente com 47 anos de idade, do sexo masculino, apresentava maxila atrófica e usava uma prótese total superior. $\mathrm{O}$ paciente foi reabilitado com quatro implantes convencionais na região anterior e dois implantes zigomáticos cônicos internos instalados nos ossos zigomáticos. Os torques de instalação dos implantes $\geq 60$ N.cm proporcionaram estabilidade primária ideal e uma prótese (protocolo) foi instalada imediatamente utilizando a técnica do assentamento passivo. O caso clínico apresentado mostrou que, após 1 ano, resultados precisos e estéticos são possíveis de alcançar com a instalação de implantes zigomáticos combinados com implantes convencionais para reabilitação de maxilas atróficas de forma imediata e sem utilização de enxerto ósseos prévios.

PALAVRAS-CHAVE: Implantes dentários; Materiais dentários; Prótese dentária; Osso zigomático, Reabilitação bucal.

\section{INTRODUÇÃO}

As perdas dentárias, traumas, infecções e pneumatização do seio maxilar torna o volume ósseo diminuído de forma acelerada e inadequado para a retenção de próteses removíveis ${ }^{1,2}$. Portanto, as reabilitações protéticas implantossuportadas de pacientes com maxilas atróficas sem aumento ósseo são desafiadoras ${ }^{2}$. A utilização de implantes zigomáticos foi introduzida por Branemark em 1988, onde foram desenvolvidos implantes com comprimentos de $30 \mathrm{~mm}$ a $52.5 \mathrm{~mm}$ com desenho para instalação no osso zigomático ${ }^{3,4}$, e tem proporcionado uma alternativa de tratamento viável à utilização de enxertos ósseos, permitindo o carregamento imediato dos implantes com próteses fixas ${ }^{2}$.
O osso da maxila é de baixa qualidade e reabsorve mais rápido do que o osso mandibular, isso resulta em dificuldades para obtenção da estabilidade primária, essencial para o carregamento imediato e a osseointegração ${ }^{5}$. A morbidade, tempo de tratamento, complicações e altos custos relacionados aos procedimentos de enxertos exigiram mudanças de paradigmas na implantodontia em relação aos conceitos, projetos e protocolos de tratamento, como carregamento imediato, implantes cônicos, implantes pterigoides e zigomáticos ${ }^{6,7}$.

O uso da porção das corticais basais para retenção dos implantes dentários em cristas reabsorvidas é um princípio ortopédico muito utilizado para instalação de implantes na maxila 
atrófica. Os implantes de comprimento mais longo facilitam o uso da bicorticalização e oferece uma estabilidade primária maior, favorecendo a instalação de uma prótese imediata ${ }^{5,8,9}$. Esse conceito pode explicar as altas taxas de sucesso com o uso de implantes zigomáticos $(94 \% \text { a } 100 \%)^{10,11}$. Portanto, os implantes zigomáticos podem fornecer uma alternativa previsível para a reabilitação de uma maxila severamente reabsorvida ${ }^{12}$, e o uso de 2 implantes zigomáticos colocados no osso zigomático (um de cada lado) combinados com implantes convencionais anteriores tem sido bastante utilizado e foi descrito com resultados favoráveis ${ }^{13,14}$. A cirurgia guiada pode facilitar o posicionamento e ancoragem dos implantes, aumentando a precisão cirúrgica. Apesar das dificuldades inerentes à angulação impulsionada da osteotomia, decorrentes do comprimento dos implantes zigomáticos, a posição correta é alcançada, de acordo com o planejado realizado no planejamento digital ${ }^{15}$.

Os implantes de conexão cônica interna apresentam o conceito de plataform switching, que tem sido amplamente indicado, apresentando características que favorecem a obtenção da estabilidade primária ideal e uma boa resposta biológica do tecido peri-implantar, como o afastamento do gap existente na interface implante/abutment do osso, a redução do componente e principalmente a diminuição do desaperto de parafusos ${ }^{16}$, que é um problema recorrente nos implantes hexágono externo ${ }^{16,17}$. O ângulo interno da superfície cônica dos implantes cônicos também influencia na redução das fraturas de parafusos e abutments devido a criação do atrito friccional ${ }^{18,19}$.

O presente artigo teve como objetivo relatar a reabilitação protética imediata de uma maxila atrófica utilizando implantes convencionais e zigomáticos com interface cônica interna como opção ao uso de enxertos ósseos prévios

\section{RELATO DE CASO CLÍNICO}

Paciente D.U., gênero masculino, 47 anos de idade, compareceu à Faculdade ILAPEO para tratamento do arco total superior desdentado. No atendimento inicial foi realizada uma avaliação do paciente, onde foram coletadas as informações de saúde geral, avaliada a oclusão, e feita uma radiografia panorâmica.

Clinicamente o paciente apresentava maxila atrofiada e arco inferior dentado total parcial (Figura 1A e 1B). A prótese total removível que o paciente fazia uso apresentava desgastes dentários e comprometia a estética do sorriso. Radiograficamente a maxila desdentada total apresentava extensa reabsorção óssea e pneumatização dos seios maxilares. Os dentes inferiores estavam em bom estado, apresentando apenas restaurações nos posteriores (Figura 2).

Foram realizadas fotografias intra e extraorais, moldagem anatômica superior e inferior, ajuste dos planos de orientação e montagem em articulador semiajustável (ASA). Em seguida, foi realizada a prova dos dentes em cera e confecção do guia multifuncional (Figura 3A e 3B). Por fim, realizou-se o registro oclusal do guia multifuncional (Figura 4) e escaneamento da maxila usando um scanner bucal (Trios, 3Shape, Copenhague, Dinamarca), que juntamente com uma tomografia sobreposta proporcionou o planejamento ideal dos implantes (Figura 5) e confecção de um guia prototipado. $\mathrm{O}$ tratamento proposto pela equipe de profissionais foi a colocação de quatro implantes convencionais e dois zigomáticos através da cirurgia guiada e uma prótese protocolo instalada imediatamente.

\section{Fase cirúrgica}

O procedimento cirúrgico foi realizado sob sedação endovenosa e anestesia infiltrativa. Inicialmente o guia cirúrgico prototipado foi posicionado e fixado com fixadores específicos. $\mathrm{O}$ preparo dos leitos ósseos foi conduzido seguindo as instruções de uso indicadas pelo fabricante, utilizando o kit cirúrgico Neodent Guided Surgery (Neodent, Curitiba, Brasil). Na região anterior as perfurações foram iniciadas pela fresa lança e em seguida com as brocas de $2.0 \mathrm{~mm}$ e $3.5 \mathrm{~mm}$. Esta conduta permitiu a instalação de quatro implantes Helix GM Acqua de 3.5 × $8 \mathrm{~mm}$ (Neodent) nas regiões dos dentes \#11, \#12, \#21 e \#22, de forma flapless. Em seguida, o guia cirúrgico foi removido e realizada incisão relaxante na região do $1^{\underline{0}}$ pré-molar e na crista óssea até a região do túber maxilar bilateral. A fresagem do osso zigomático foi realizada bilateral, utilizando a sequência de brocas indicadas pelo fabricante $(2.35 \mathrm{~mm}$, piloto $2.3 / 3.2 \mathrm{~mm}, 3.75 \mathrm{~mm}$ e $4.0 \mathrm{~mm}$ ) com o guia em posição. O preparo final foi realizado com a broca multilaminada, afastamento da membrana dos seios maxilares, colocação de biomaterial de origem bovina Botiss Cerabone ${ }^{\circledR}$ (Botiss biomaterials $\mathrm{GmbH}$, Zossen, Alemanha) e por fim, instalação de dois implantes Zygoma GM $^{\mathrm{TM}}$ Neoporos de 4.0 x $42.5 \mathrm{~mm}$ (Neodent) (Figura 6A e 6B). Todos os implantes foram instalados com torques $\geq 60 \mathrm{~N}$.cm, permitindo a realização da provisionalização imediata através de uma prótese total (protocolo). Foram instalados dois mini pilares cônicos GM angulados de 45 graus com transmucoso de $2.5 \mathrm{~mm}$ e $3.5 \mathrm{~mm}$ (Neodent) sobre os implantes zigomáticos das regiões dos dentes \#16 e \#26, respectivamente. Na região anterior foram instalados dois Micro pilares GM (Neodent) (dentes \#11 e \#21) e dois mini pilares cônicos GM (Neodent) (dentes \#12 e \#22), todos com transmucoso de $2.5 \mathrm{~mm}$. Em seguida, uma membrana de origem porcina Jason ${ }^{\circledR}$ (Botiss) foi utilizada para recobrimento vestibular dos implantes zigomáticos e a maxila suturada.

\section{Fase protética}

Em seguida, os transferentes de moldeira aberta (Neodent) foram posicionados sobre os mini pilares e micro pilares e unidos ao guia multifuncional em oclusão com o arco inferior utilizando resina acrílica Pattern (GC America Inc, Alsip, USA). A moldagem foi realizada com silicone de condensação Speedex (Coltene, Altstätten, Suíça) e enviada ao laboratório de prótese. No dia seguinte, foi realizada prova dos dentes em cera e barra (Figura 7A). A prótese acrílica híbrida foi realizada de acordo com a técnica do assentamento passivo e instalada quatro dias após a cirurgia. Por fim, os ajustes oclusais foram realizados, aliviando os contatos excessivos nos movimentos excursivos (Figuras 7B e 7C).

O paciente passou por acompanhamentos periódicos para avaliação clínica e radiográfica da reabilitação. Clinicamente, foi possível observar uma remodelação mínima dos tecidos peri-implantares e radiograficamente boa manutenção do osso marginal após 1 ano (Figura 8A a 8C).

\section{DISCUSSÃO}

Neste relato de caso, descrevemos a reabilitação de uma maxila atrófica com dois implantes zigomáticos instalados nos ossos zigomáticos e quatro implantes convencionais cônicos internos instalados na região anterior e vários fatores influenciaram na decisão. 


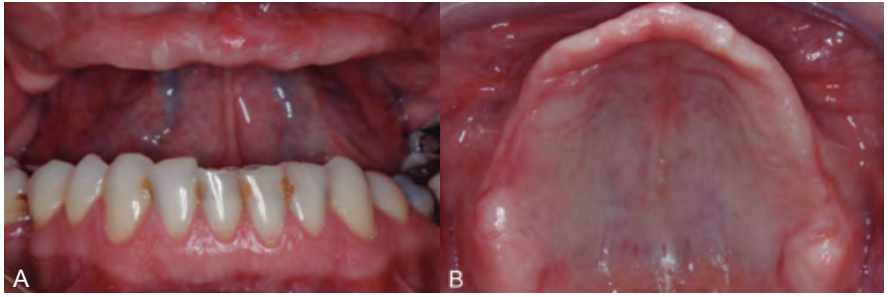

Figura 1 - Aspecto inicial do caso clínico. (A) Vista frontal; (B) Vista oclusal da maxila atrófica.

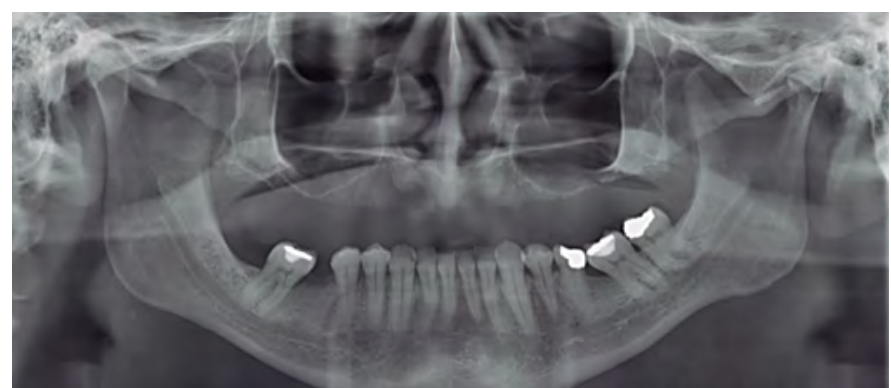

Figura 2 - Radiografia panorâmica inicial.

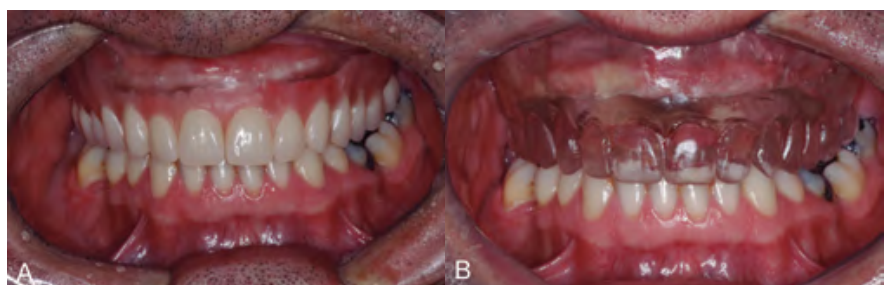

Figura 3 - Parte protética inicial. (A) Prova dos dentes em cera; (B) Prova do guia multifuncional.

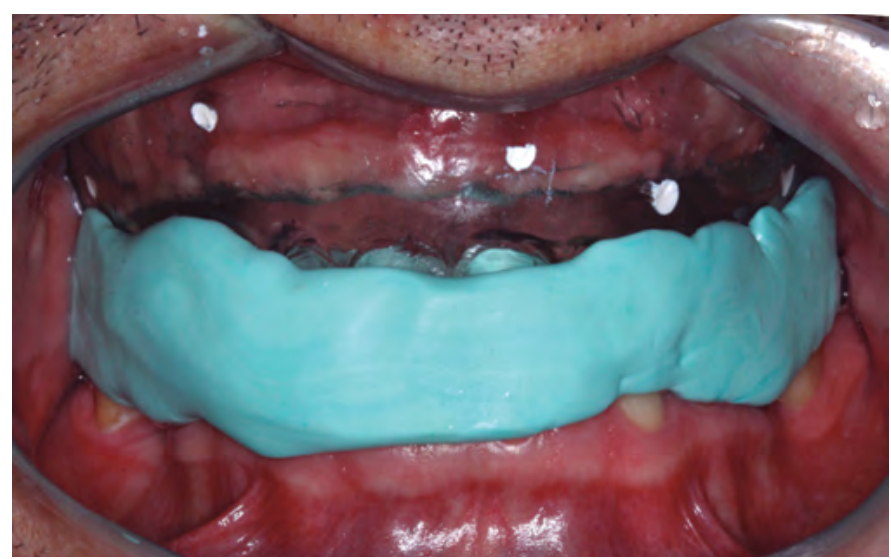

Figura 4 - Registro oclusal e marcação dos pontos em guta-percha para realização da tomografia e escaneamento.

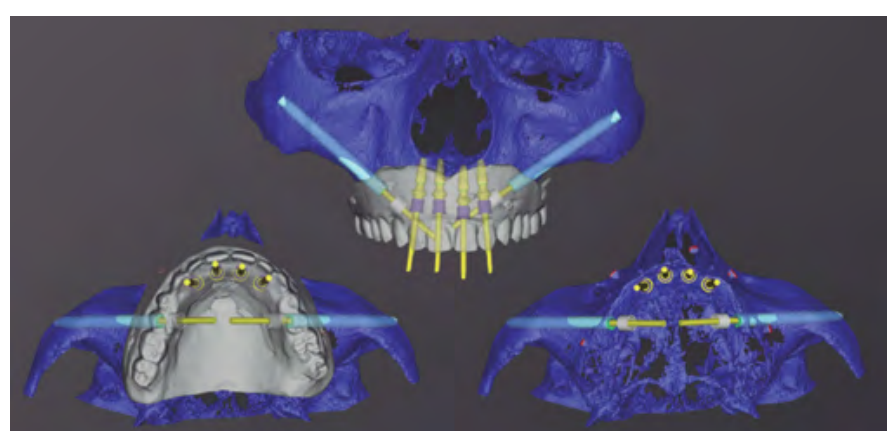

Figura 5 - Planejamento digital dos implantes.
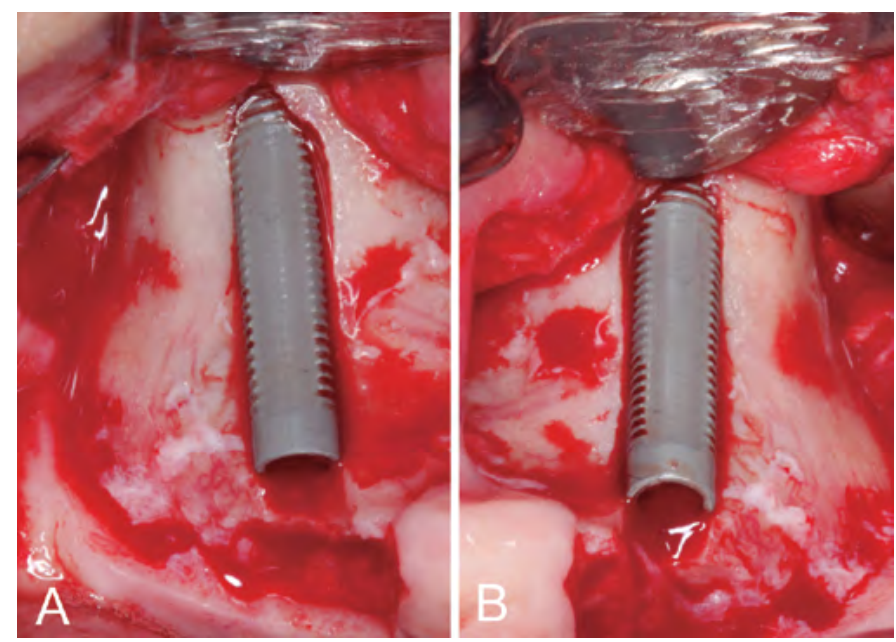

Figura 6 - Parte cirúrgica. (A) Instalação do implante zigomático na região do dente \#16; (B) Instalação do implante zigomático na região do dente \#26.

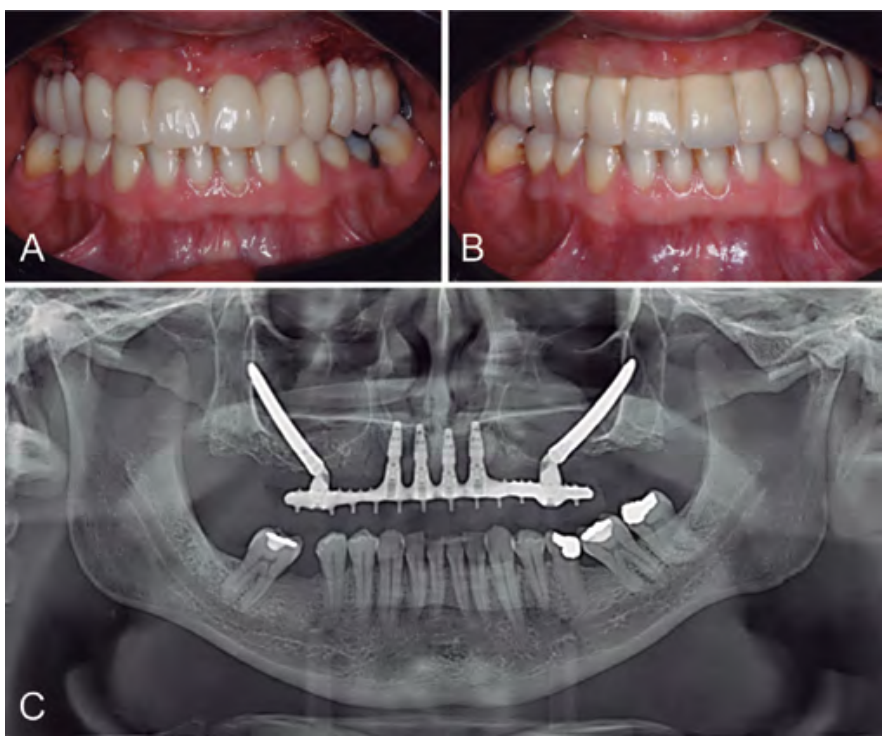

Figura 7 - Parte protética final. (A) Prova dos dentes em cera; (B) Prótese protocolo instalada imediata e após ajustes oclusais; (C) Radiografia panorâmica do pós-operatório imediato.

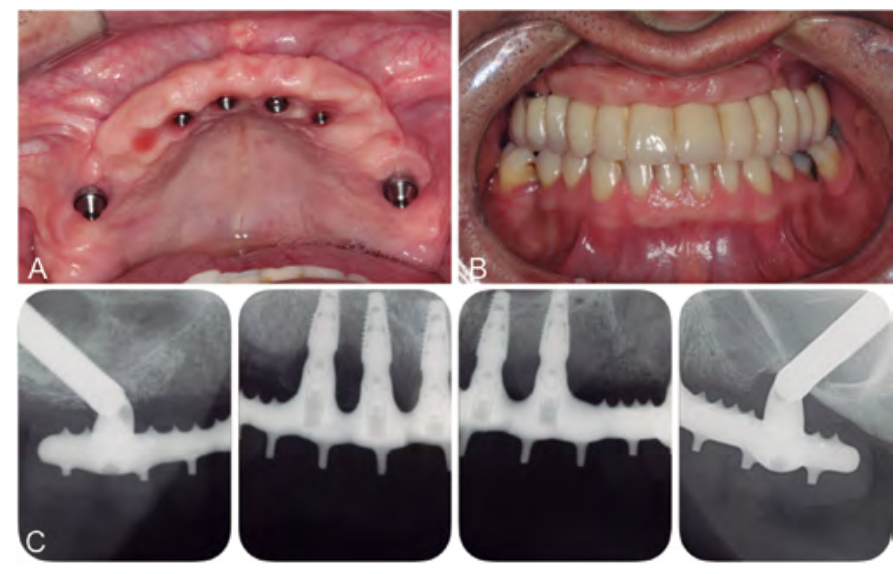

Figura 8 - Acompanhamento de 1 ano. (A) Aspecto clínico dos tecidos moles; (B) Sorriso do paciente; (C) Radiografias periapicais da reabilitação. 
Inicialmente, não era possível instalar implantes convencionais na região posterior da maxila por falta de disponibilidade óssea adequada e devido ao paciente não desejar passar por procedimentos de enxertia óssea prévia por receio de uma possível perda do enxerto, além do tempo de espera para instalação dos implantes. Entretanto, apesar da atrofia maxilar severa, o paciente apresentava disponibilidade óssea necessária para a colocação de dois implantes zigomáticos (um de cada lado) nos ossos zigomáticos. Além disso, a técnica já foi descrita anteriormente com sucesso ${ }^{10}$.

A cirurgia em dois tempos cirúrgicos gera um maior estresse para o paciente devido o número de procedimentos realizados, além de que a técnica não impede o colapso dos tecidos. Nesses casos podem ser necessários procedimentos de enxertia posterior para conseguir recuperar o volume tecidual perdido na remodelação que acontece após a instalação dos implantes ${ }^{20}$. Existem evidências científicas afirmando que a instalação do implante e carregamento imediato favorecem a manutenção do volume tecidual pelo micro estímulo gerado no osso ${ }^{20}$. Outro fator importante é a precisão da fresagem do osso, para obter uma estabilidade primária ideal, por isso existem alguns desenhos de implantes com o objetivo de se conseguir estabilidade primária em osso de diferentes qualidades ${ }^{21,22}$. A instalação dos implantes infra-ósseos também é um fator que influencia positivamente na manutenção dos tecidos peri-implantares ${ }^{21,23}$.

Em estudos passados foram relatados resultados previsíveis após a colocação de dois implantes zigomáticos associados a implantes convencionais anteriores ${ }^{10,24}$. No estudo atual, o sucesso dos implantes zigomáticos relatados está de acordo com as altas taxas de sucesso descritas anteriormente ${ }^{10,11,24}$. Os valores de torque $\geq 60$ N.cm que foram obtidos durante a colocação dos implantes permitiu a aplicação da técnica de carregamento imediato $^{3}$. Outros estudos também relataram bons resultados com o carregamento imediato de implantes zigomáticos para reabilitação de maxilas atróficas e propuseram que a densidade óssea do osso zigomático proporciona o travamento ideal para o uso do carregamento protético imediato ${ }^{12,14,25}$. Portanto, o sucesso da técnica de reabilitação com implantes zigomáticos está relacionado aos conceitos biomecânicos.

O planejamento por software e cirurgias guiadas combinado a implantes com macrogeometria favorável a realização da carga imediata é importante para reabilitações unitárias ou múltiplas satisfatórias para os pacientes ${ }^{21,23,26,27}$. A técnica da cirurgia guiada pode ser utilizada para fresagem e instalação dos implantes ${ }^{26}$, ou apenas para fresagem, sendo os implantes instalados com mãos livres ${ }^{27}$, ficando a critério do cirurgião-dentista a escolha da técnica. A cirurgia guiada apresenta vantagens como o posicionamento adequado do implante como planejado no software e menor morbidade ao paciente ${ }^{21,27}$. A macrogeometria do implante e o tipo de conexão influenciam no valor de torque ideal para carga imediata e na perda óssea peri-implantar ${ }^{21,27,29}$.

Outros fatores foram importantes para resolução do caso clínico apresentado, a utilização de mini pilares cônicos e micro pilares para os implantes convencionais e angulados de 45 graus para os zigomáticos promoveram a saída dos parafusos na oclusal dos dentes da prótese, além de que a técnica do assentamento passivo proporcionou o assentamento correto da prótese sobre os abutments ${ }^{3,28}$.
Para o preenchimento dos seios maxilares e sobre implantes zigomáticos substitutos ósseos do tipo xenógeno tem sido indicado para manter o volume do osso alveolar ${ }^{29,30}$. Neste caso relatado, o preenchimento dos seios maxilares com o osso de origem bovina foi importante para evitar o contato dos implantes zigomáticos com a membrana de Schneider. Portanto, mesmo que o osso autógeno continue sendo o padrão ouro, é possível a obtenção de resultados altamente favoráveis com o uso dos biomateriais, sem precisar de mais um leito cirúrgico ${ }^{20}$. Por fim, a membrana porcina utilizada recobriu os implantes zigomáticos, evitando o contato íntimo com os tecidos moles.

Taxas mais elevadas de satisfação relacionadas ao uso de implantes zigomáticos foram descritas anteriormente ${ }^{14}$. Nesse relato de caso, não houve complicações associadas à colocação dos implantes zigomáticos ou ao uso do protocolo de carregamento imediato. Além disso, o paciente relatou satisfação com a reabilitação, associando a uma melhoria na qualidade de vida, considerando que a reabilitação foi eficaz e evitou procedimentos mais invasivos e extensos, como enxertos ósseos autógenos de locais doadores extraorais.

\section{CONCLUSÃO}

O caso clínico apresentado mostrou que a instalação de dois implantes zigomáticos com interface cônica interna, combinados com implantes convencionais e carregamento protético imediato é uma técnica segura e previsível para o tratamento de maxilas atróficas, sendo uma excelente alternativa aos procedimentos de enxerto ósseo. No entanto, a instalação dos implantes zigomáticos é um procedimento cirúrgico complexo e exige cirurgiões experientes, considerando a presença de algumas estruturas anatômicas importantes envolvidas.

\section{REFERÊNCIAS}

01. Adell R, Lekholm U, Rockler B, Brånemark PI. A 15-year study of osseointegrated implants in the treatment of the edentulous jaw. Int J Oral Surg. 1981; 10(6): 387-416.

02. Petrungaro PS, Gonzales S, Villegas C, Yousef J, Arango A. A retrospective study of a multi-center case series of 452 zygomatic implants placed over 5 years for treatment of severe maxillary atrophy. Compend Contin Educ Dent. 2020; 41(4): 232-241.

03. Padovan LE, Ribeiro-Júnior PD, Sartori IAM, Thomé G, Sartori EM, Uhlendorf J. Multiple zygomatic implants as an alternative for rehabilitation of the extremely atrophic maxilla: a case letter with 55 months of follow-up. J Oral Implantol. 2015; 41(1): 97-100.

04. Stiévenart M, Malevez C. Rehabilitation of totally atrophied maxilla by means of four zygomatic implants and fixed prosthesis: a 6-40-month follow-up. Int J Oral Maxillofac Surg. 2010; 39(4): 358-363.

05. Agliardi EL, Romeo D, Panigatti S, Nobre MA, Maló P. Immediate full-arch rehabilitation of the severely atrophic maxilla supported by zygomatic implants: a prospective clinical study with minimum follow-up of 6 years. Int J Oral Maxillofac Surg. 2017; 46(12): 1592-1599.

06. Odin G, Mish CE, Binderman I, Scortecci G. Fixed rehabilitation of severely atrophic jaws using immediately loaded basal disk implants after in situ bone activation. J Oral Implantol. 2012; 38(5): 611-616.

07. Preshaw P. Summary of: implant surface characteristics and their effect on osseointegration. Br Dent J. 2015; 218(5): 292-293.

08. Garg R, Mishra N, Alexander M, Gupta SK. Implant survival between endo-osseous dental implants in immediate loading, 
delayed loading, and basal immediate loading dental implants a 3-year follow-up. Ann Maxillofac Surg. 2017; 7(2): 237-244.

09. Hsu A, Seong WJ, Wolff R, Zhang L, Hodges J, Olin PS, et al. Comparison of initial implant stability of implants placed using bicortical fixation, indirect sinus elevation, and unicortical fixation. Int J Oral Maxillofac Implants. 2016; 31(2): 459-468.

10. Chrcanovic BR, Abreu MH. Survival and complications of zygomatic implants: a systematic review. Oral Maxillofac Surg. 2013; 17(2): 81-93.

11. Urgell JP, Gutiérrez VR, Escoda CGG. Rehabilitation of atrophic maxilla: a review of 101 zygomatic implants. Med Oral Patol Oral Cir Bucal. 2008; 13(6): E363-E370.

12. Maló P, Nobre MD, Lopes A, Francischone C, Rigolizzo M. Threeyear outcome of a retrospective cohort study on the rehabilitation of completely edentulous atrophic maxillae with immediately loaded extra-maxillary zygomatic implants. Eur J Oral Implantol. 2012; 5(1): $37-46$

13. Davó R, Malevez C, Rojas J, Rodriguez J, Regolf J. Clinical outcome of 42 patients treated with 81 immediately loaded zygomatic implants: a 12- to 42-month retrospective study. Eur J Oral Implantol. 2008; 1(2): 141-150.

14. Sartori EM, Padovan LEM, Sartori IAM, Ribeiro Junior PD, Carvalho ACGS, Goiato MC. Evaluation of satisfaction of patients rehabilitated with zygomatic fixtures. J Oral Maxillofac Surg. 2012; 70(2): 314-319.

15. Schiroli G, Angiero F, Silvestrini-Biavati A, Benedicenti S. Zygomatic implant placement with flapless computer-guided surgery: a proposed clinical protocol. J Oral Maxillofac Surg. 2011; 69(12): 2979-2989.

16. Castro DS, Araujo MA, Benfatti CA, Araujo CR, Piatelli A, Perrotti V, et al. Comparative histological and histomorphometrical evaluation of marginal bone resorption around external hexagon and Morse cone implants: an experimental study in dogs. Implant Dent. 2014; 23(3): 270-276.

17. Moura MB, Rodrigues RB, Pinto LM, Araújo CA, Novais VR, Simamoto Júnior PC. Influence of screw surface treatment on retention of implant-supported fixed partial dentures. J Oral Implantol. 2017; 43(4): 254-260.

18. Verdugo CL, Núñez GJ, Avila AA, San Martín CL. Microleakage of the prosthetic abutment/implant interface with internal and external connection: in vitro study. Clin Oral Implants Res. 2014; 25(9): 1078-1083.

19. Moura MB, Loureiro KRT, Lima LB, Felippi C, Júnior PCS. Biomechanical behavior of three different types of internal tapered connectios after cyclic and static loading tests: experimental in vitro. Int J Implant Dent. 2020; 6(1): 41.
20. Maló P, Araújo Nobre M, Lopes A, Rodriguez R. Preliminary report on the outcome of tilted implants with longer lengths $(20-25 \mathrm{~mm})$ in low-density bone: one-year follow-up of a prospective cohort study. Clin Implant Dent Relat Res 2015; 17(suppl.1): 134-42.

21. Thomé G, Pires R, Sallati RC, Cartelli CA, Moura MB, Trojan LC. Insertion torque of hybrid tapered implants with different types of instrumentation: an in vitro analysis and report of two cases. J Osseointegr. 2019; 11(2): 98-106.

22. Cha HS, Kim JW, Hwang JH, Ahn KM. Frequency of bone graft in implant surgery. Maxillofac Plast Reconstr Surg. 2016; 38(1): 19.

23. Reyes AJS, Thomé G, Cartelli CA, Bernardes SR, Moura MB, Trojan LC. Previsibilidade na instalação imediata de implante cone Morse pelo planejamento digital e provisionalização imediata: Relato de caso. Rev Odontol Bras Central. 2019; 28(85): 77-81.

24. Migliorança RM, Coppedê A, Rezende RCD, Mayo T. Restoration of the edentulous maxilla using extrasinus zygomatic implants combined with anterior conventional implants: a retrospective study. Int J Oral Maxillofac Implants. 2011; 26(3): 665-672.

25. Maló P, Nobre MA, Lopes I. A new approach to rehabilitate the severely atrophic maxilla using extramaxillary anchored implants in immediate function: a pilot study. J Prosthet Dent. 2008; 100(5): 354-366.

26. Thomé G, Moura MB, Cartelli CA, Uhlendorf J, Trojan LC, Vitório MSKD, et al. Posicionamento protético ideal na zona estética de acordo com o fluxo de trabalho digital. Prótese News. 2020; 7(1): 54-63.

27. Thomé G, Trojan LC, Bernardes SR, Cartelli CA, Uhlendorf J, Moura MB. Guided osteotomy and implants placed with free hands for double full arch rehabilitation with immediate prosthetic loading. Rev Gaúch Odontol. 2020; 68: e20200025.

28. Able FB, Sartori IAM, Thomé G, Melo ACM. Retrospective, cross-sectional study on immediately loaded implant-supported mandibular fixed complete-arch prostheses fabricated with the passive fit cementation technique. J Prosthet Dent. 2018; 119(1): 60-66.

29. Martin C, Thomé G, Melo AC, Fontão FN. Peri-implant bone response following immediate implants placed in the esthetic zone and with immediate provisionalization -- a case series study. Oral Maxillofac Surg. 2015; 19(2): 157-163.

30. Han CH, Mangano F, Mortellaro C, Park KB. Immediate loading of tapered implants placed in postextraction sockets and healed sites. J Craniofac Surg. 2016; 27(5): 1220-1227.

\section{ABSTRACT}

The rehabilitation of atrophic upper jaws with dental implants is always a challenge, since the use of bone grafts is almost always necessary to stabilize the implants. Zygomatic implants have been used as a viable option for the use of bone grafting procedures prior to surgery. The internal tapered interface presents a reduced gap between implant and prosthetic component, showing satisfactory long-term biological and aesthetic results. This article aims to report the immediate prosthetic rehabilitation of an atrophic upper jaw with conventional and zygomatic implants with an internal tapered interface as an option for the use of previous bone grafts. A 47-year-old male patient presented with an atrophic upper jaw and used an upper total prosthesis. The patient was rehabilitated with four conventional implants in the anterior region and two internal tapered zygomatic implants placed in the zygomatic bones. The implant placement torques $\geq$ 60 N.cm provided ideal primary stability and a prosthesis (protocol) was installed immediately using the passive fit technique. The clinical case presented showed that, after 1 year, accurate and aesthetic results are possible to achieve with the placement of zygomatic implants combined with conventional implants for 
the rehabilitation of atrophic upper jaws immediately and without the use of previous bone grafts.
KEYWORDS: Dental implants; Dental materials; Dental prothesis; Zygomatic bone; Oral Rehabilitation.

\section{AUTOR PARA CORRESPONDÊNCIA}

Marcos Boaventura de Moura

Departamento de Implantodontia

Instituto Latino Americano de Pesquisa e Ensino

Odontológico (ILAPEO)

Rua Jacarezinho, 656, CEP: 80710-150, Curitiba-PR, Brasil

Telefone/Fax: (41) 35956000

E-mail: boaventura.mm@hotmail.com 